\title{
Subperiosteal hemorrhage due to a distal femoral physeal fracture in a neonate
}

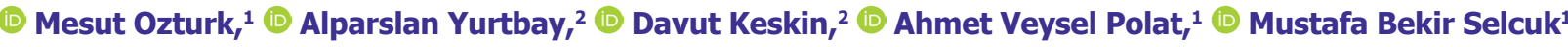 \\ ${ }^{1}$ Department of Radiology, Ondokuz Mayis University Faculty of Medicine, Samsun, Turkey \\ ${ }^{2}$ Department of Orthopedics and Traumatology, Ondokuz Mayis University Faculty of Medicine, Samsun, Turkey
}

\begin{abstract}
Femoral physeal fractures have been rarely reported as a birth-related injury. As the plain radiograph findings are variable, the diagnosis may be challenging. In this case report, we describe a male neonate presenting with periosteal elevation at the left distal femur. A radiological evaluation demonstrated posteromedial displacement of the distal femoral epiphysis. The final diagnosis was subperiosteal hemorrhage due to a distal femoral physeal fracture.

Keywords: Birth injuries; distal femur; neonate; subperiosteal hemorrhage; physeal fracture.

Cite this article as: Ozturk M, Yurtbay A, Keskin D, Polat AV, Selcuk MB. Subperiosteal hemorrhage due to a distal femoral physeal fracture in a neonate. North Clin Istanb 2019;6(3):312-314.
\end{abstract}

$\mathrm{B}$ irth-related limb injuries have been rarely reported. This spectrum includes simple bruises, swelling, forceps scars, loss of the nerve and motor function, or rarely a fracture [1]. Injuries related to the femur are usually found at the shaft and proximal physis, but distal femoral physeal fractures are rarely reported [1-3]. Herein, we reported a male newborn with the periosteal elevation at the left distal femur. Magnetic resonance imaging (MRI) demonstrated a distal femoral physeal fracture.

\section{CASE REPORT}

An informed consent was obtained for this case presentation. A 13-day-old male neonate was referred to our health center with the complaint of swelling and immobility in his left thigh. He was born at the $40^{\text {th }}$ gestational week by a vaginal delivery with an APGAR score of 10 . He was appropriate for the gestational age, and his birth weight was $3400 \mathrm{~g}$. An initial physical examination was unremarkable. The obstetrician reported no complications related to delivery. The mother attended her follow- ups regularly during the pregnancy, and the pregnancy was uneventful.

At the $2^{\text {nd }}$ day following birth, the mother noticed soft tissue swelling around the patient's left knee and distal femur. On physical examination, there was slight swelling around the left knee and distal femur. Femoral and dorsal pedal pulses were palpable. The range of motion at the knee joint was normal without any evidence of joint instability or dislocation. His white blood cell count was $11,090 / \mu \mathrm{L}$, the hemoglobin level $14.2 \mathrm{~g} / \mathrm{dl}$, platelet count $629,000 / \mu \mathrm{L}$, and serum $\mathrm{C}$-reactive protein level $<1 \mathrm{mg} / \mathrm{L}$. Coagulation parameters including the prothrombin time and activated partial thromboplastin time were also within the normal range. Plain radiograph of the region was normal. The patient started to receive antibiotic treatment for 10 days with a preliminary diagnosis of soft tissue infection. After the antibiotherapy, his swelling around the left distal femur was still present. A repeated radiograph of the left thigh demonstrated a periosteal reaction around the left distal femur (Fig. 1). No fracture line could be identified, but a slight poste-

Received: December 31, 2017 Accepted: April 13, 2018 Online: August 08, 2018

Correspondence: Dr. Mesut OZTURK. Ondokuz Mayis Universitesi Tip Fakultesi, Radyoloji Anabilim Dali, 55132 Samsun, Turkey. Phone: +90 3623121919 - 2541 e-mail: dr.mesutozturk@gmail.com

(c) Copyright 2019 by Istanbul Provincial Directorate of Health - Available online at www.northclinist.com 

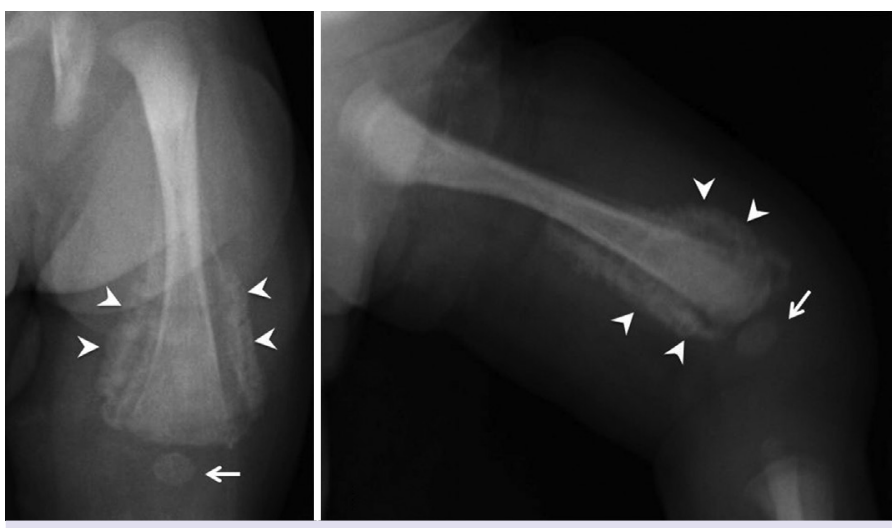

FIGURE 1. A radiograph of the left femur and knee demonstrates periosteal reaction (arrowheads) and soft tissue swelling at the left distal femur. Slight posterior displacement of the femoral epiphyseal ossification center is visible (arrow). No fracture line is identified.

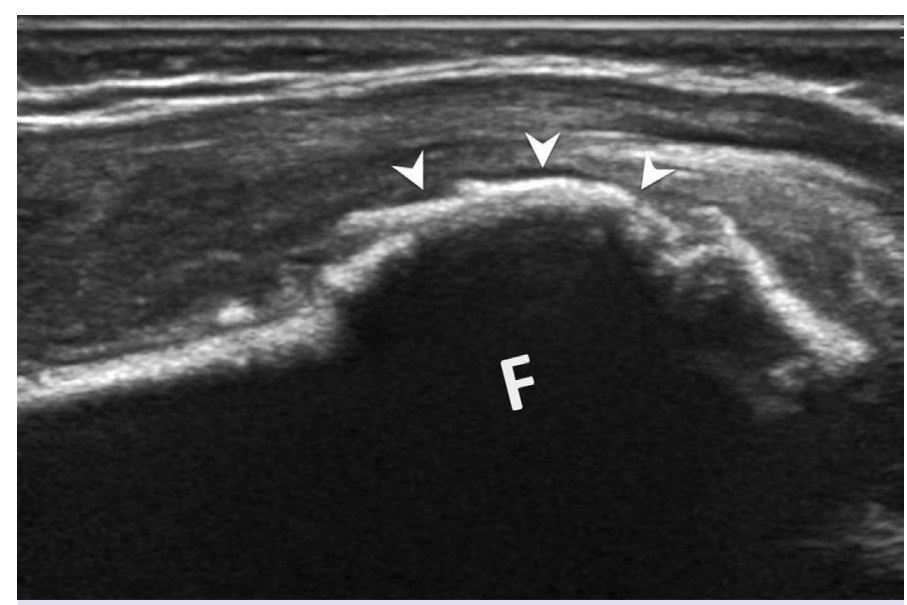

FIGURE 2. Sagittal ultrasound image of the left distal femur shows a calcified periosteal elevation (arrowheads) at the distal femur (F).

rior displacement of the distal femoral epiphysis was suspected. Further investigation with ultrasound (US) demonstrated a calcified periosteal elevation (Fig. 2). The patient underwent an MRI, which demonstrated posterior displacement of the distal femoral epiphysis (Fig. 3). A femoral physeal fracture and subperiosteal hemorrhage was the final diagnosis.

Serum vitamin $C$ levels in both the patient and his mother were tested to rule out neonatal scurvy, and they were within the normal range. A skeletal survey was performed to rule out a non-accidental trauma, and no other fractures were identified. Metabolic tests for osteogenesis imperfecta were negative. The family was advised that

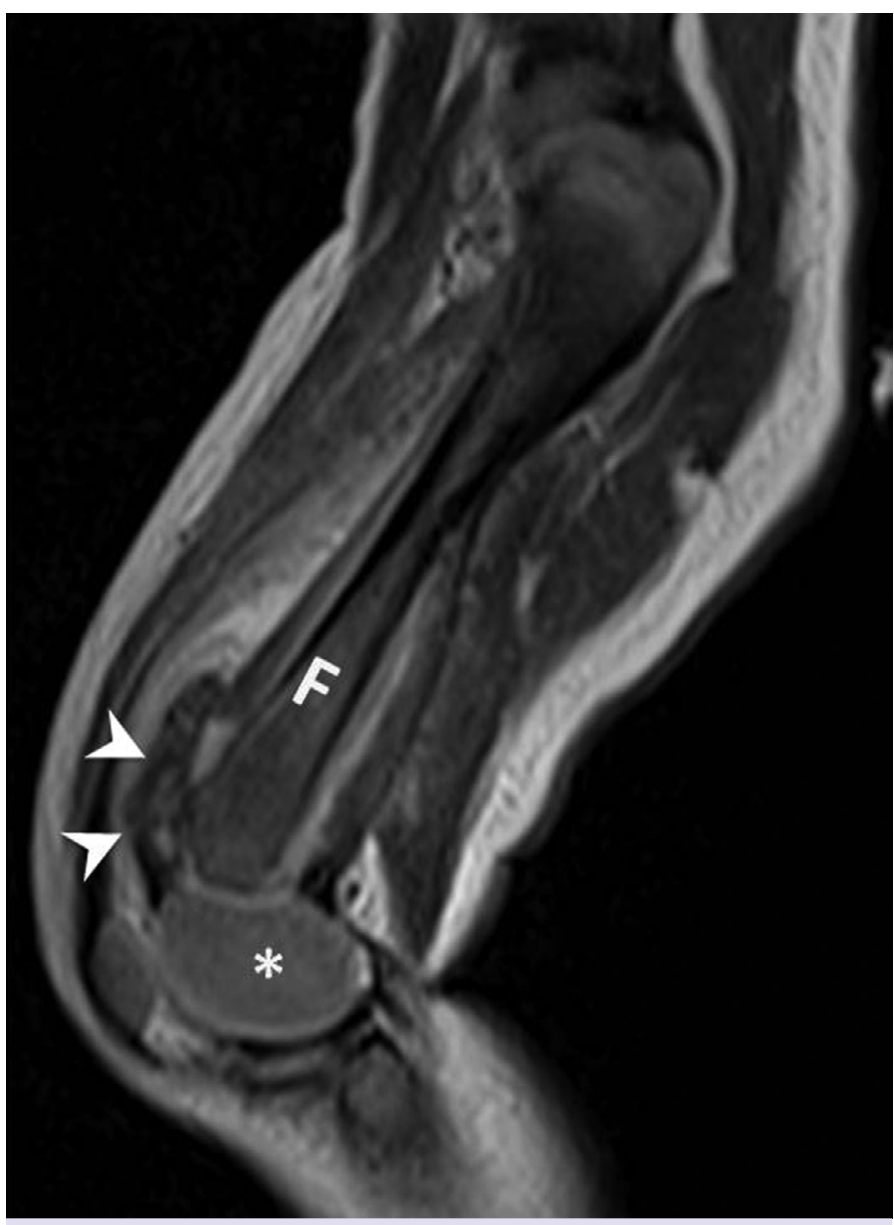

FIGURE 3. Sagittal T2-weighted magnetic resonance imaging of the left thigh shows posterior displacement of the distal physeal (asterix) and periosteal elevation (arrowheads) around the distal femur (F).

the fracture was already in the healing process, and no active therapeutic intervention was needed. The infant was discharged and closely followed up. The follow-up plain radiograph performed 3 weeks later demonstrated a periosteal new bone formation at the distal femur (Fig. 4).

\section{DISCUSSION}

A distal femoral physeal fracture is a rare birth-related injury causing subperiosteal hemorrhage in newborns. It may occur after a difficult vaginal or cesarean delivery [2, 3]. Traumatic femoral physeal fractures are usually associated with a sudden, forced traction of the limb, with acute angulation or twisting. The force causes rupture at the epiphyseal attachment of the periosteum, where is the weakest portion [2]. A tear in the periosteum results in subperiosteal hemorrhage, which gradually calcifies and 


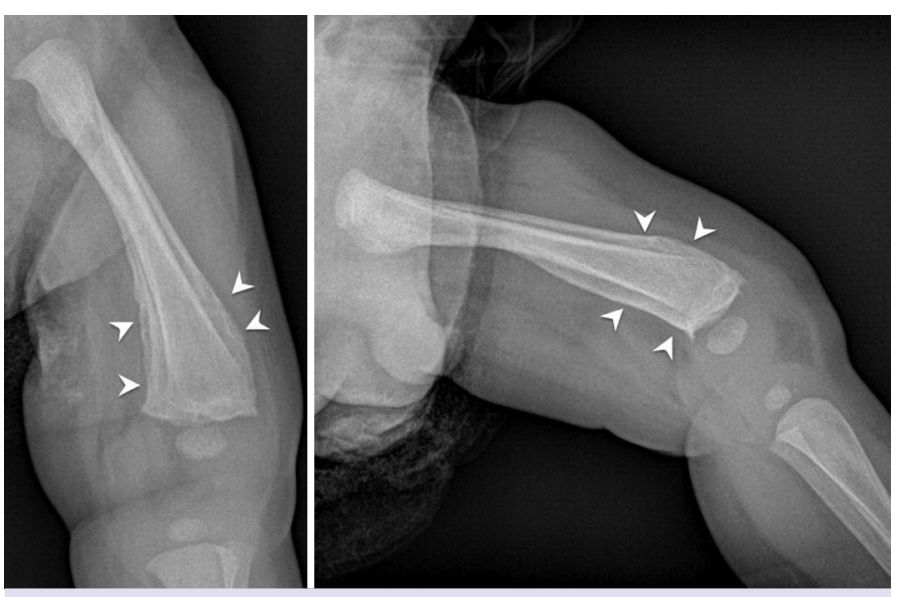

FIGURE 4. Follow-up plain radiograph of the left femur shows periosteal new bone formation (arrowheads).

results in callus and new subperiosteal bone formation. Infants present with swelling, erythema, and tenderness at the affected area. It is often mistaken for septic arthritis or osteomyelitis, and many patients receive antibiotics [3]. Coexistence of the subperiosteal hemorrhage with an epiphyseal fracture is important to exclude other causes of subperiosteal hemorrhage such as infection, tumor, or scurvy [4].

Initial radiographs of a physeal fracture in a neonate may demonstrate soft tissue swelling, but if the physis is not ossified, they may reveal no bone abnormality. Plain radiograph findings include epiphyseal displacement, widening of the physis, and haziness of the smooth margins of the metaphysis and epiphysis [5]. MRI and US proved to be useful in making the correct diagnosis $[3,6,7]$. They may show the periosteal elevation around the distal femur and displacement of the epiphysis. If the periosteal reaction calcifies, an acoustic shadow due to calcification may obscure the fracture. Follow-up radiographs can confirm the diagnosis by showing a periosteal reaction and new bone formation at the injured site [5]. Treatment strategies differ widely, from an uneventful spontaneous healing process to a closed reduction [8].

An initial evaluation of our patient was in another local hospital, and the initial radiograph was evaluated as normal by a pediatrician. There was no history of any birth-related complication, so a physeal fracture was not considered in the differential list, and the patient received antibiotics for a preliminary diagnosis of a soft tissue in- fection. After the patient did not respond to the medical therapy and the immobility of the joint was noticed, he was re-evaluated in our center. A combined interpretation of the plain radiograph and MRI of the region demonstrated a distal femoral physeal fracture.

As the physeal fractures are rare, and their radiological findings are variable, they present a diagnostic challenge in newborns. Physeal fractures should be considered in the differential list in any neonate presenting with a limb swelling. Thus, invasive procedures such as joint aspiration for diagnostic purposes or unnecessary use of antibiotics can be avoided. An MRI is very helpful in making the correct diagnosis. Pediatricians, orthopedists, and radiologists dealing with newborns should be familiar with this rare condition.

Informed Consent: Written informed consent was obtained from the patient for the publication of the case report and the accompanying images.

Conflict of Interest: The authors declare that they have no conflict of interest.

Financial Disclosure: The authors declared that this study has received no financial support.

Authorship Contributions: Concept - MO, AY; Design - MO, AY; Supervision - MO, AY, DK, AVP, MBS; Materials - MO, AY, DK, AVP, MBS; Data collection \&/or processing - MO, AY; Analysis and/or interpretation - MO, AY; Writing - MO, AY, DK, AVP, MBS.

\section{REFERENCES}

1. Kancherla R, Sankineani SR, Naranje S, Rijal L, Kumar R, Ansari T, et al. Birth-related femoral fracture in newborns: risk factors and management. J Child Orthop 2012;6:177-80. [CrossRef]

2. Rutherford Y, Fomufod AK, Gopalakrishnan LJ, Beeks EC. Traumatic distal femoral periostitis of the newborn: a breech delivery birth injury. J Natl Med Assoc 1983;75:933-5.

3. Jain R, Bielski RJ. Fracture of lower femoral epiphysis in an infant at birth: a rare obstetrical injury. J Perinatol 2001;21:550-2. [CrossRef]

4. Polat AV, Bekci T, Say F, Bolukbas E, Selcuk MB. Osteoskeletal manifestations of scurvy: MRI and ultrasound findings. Skeletal Radiol 2015;44:1161-4. [CrossRef]

5. Rogers LF, Poznanski AK. Imaging of epiphyseal injuries. Radiology 1994;191:297-308. [CrossRef]

6. White PG, Mah JY, Friedman L. Magnetic resonance imaging in acute physeal injuries. Skeletal Radiol 1994;23:627-31. [CrossRef]

7. Carey J, Spence L, Blickman H, Eustace S. MRI of pediatric growth plate injury: correlation with plain film radiographs and clinical outcome. Skeletal Radiol 1998;27:250-5. [CrossRef]

8. Kamaci S, Danisman M, Marangoz S. Neonatal physeal separation of distal humerus during cesarean section. Am J Orthop (Belle Mead NJ) 2014;43:E279-81. 